\title{
PUNICA GRANATUM RIND EXTRACT: ANTIBIOTIC POTENTIATOR AND EFFLUX PUMP INHIBITOR OF MULTIDRUG RESISTANT KLEBSIELLA PNEUMONIAE CLINICAL ISOLATES
}

\author{
ZUMAANA RAFIQ ${ }^{1}$, SREEVIDYA NARASIMHAN ${ }^{1}$, MAGESH HARIDOSS ${ }^{1}$, ROSY VENNILA ${ }^{2}$, RAMA VAIDYANATHAN ${ }^{1 *}$ \\ ${ }^{1}$ Department of Biotechnology, Dr. M.G.R. Educational and Research Institute, E.V.R. Periyar Salai, Maduravoyal, Chennai, Tamil Nadu, India. \\ ${ }^{2}$ Department of Microbiology, Institute of Surgical Gastroenterology and Liver Transplantation, Government Stanley Medical College and \\ Hospital, Chennai, Tamil Nadu, India. Email: ramavaidy@gmail.com
}

Received: 02 November 2016, Revised and Accepted: 23 November 2016

ABSTRACT

Objective: With a rise in multidrug resistant (MDR) bacterial isolates, search for antibiotics or compounds that could act synergistically with them is a significant area of research. Efflux-mediated resistance, in particular, is a great hurdle that needs to be overcome. In an effort to identify such synergistic compounds and potential efflux pump inhibitors (EPI), we analyzed the rind of Punica granatum (pomegranate) against MDR clinical Klebsiella pneumoniae isolates.

Methods: Sequential fractionation of P. granatum rind ethanol (PGR) extract was carried out to obtain hexane, butanol and water fractions. Antibacterial activity of the plant extracts was confirmed, and synergistic interaction with antibiotics was determined by the checkerboard assay. Gas chromatography-mass spectrometry (GC-MS) analysis was performed to identify the phytochemical constituents of the hexane extract. To study EPI activity of the extracts, norfloxacin accumulation assay was carried out.

Results: PGR ethanol extract was found to have synergistic activity with ciprofloxacin, levofloxacin, ceftazidime, cefoxitin, meropenem, and gentamicin resulting in fold decrease of minimum inhibitory concentration (MIC) ranging from 2 to 32 fold. The hexane fraction was found to have maximum synergistic activity resulting in a 32-fold reduction of ciprofloxacin MIC followed by butanol and water fractions. The PGR ethanol extract was also found to have efflux inhibition activity by the norfloxacin accumulation assay. Of the sequential fractions, the butanol fraction had maximum efflux inhibition activity.

Conclusion: Therefore, our study shows that PGR extract can potentiate the effect of antibiotics on MDR bacteria, and the mode of action is likely to be due to EPI.

Keywords: Punica granatum rind, Pomegranate, Synergy with antibiotics, Multidrug resistant, Klebsiella pneumoniae, Efflux pump inhibition.

(C) 2017 The Authors. Published by Innovare Academic Sciences Pvt Ltd. This is an open access article under the CC BY license (http://creativecommons. org/licenses/by/4. 0/) DOI: http://dx.doi.org/10.22159/ajpcr.2017.v10i3.16000

\section{INTRODUCTION}

Multidrug resistance (MDR) is increasing worldwide at an alarming rate [1]. To combat this, stronger antibiotics in higher dosages are prescribed, thereby resulting in a vicious cycle of further resistance. Klebsiella pneumoniae, an opportunistic pathogen, causes numerous diseases like Pneumonia, bloodstream infections, wound or surgical site infections, urinary tract infections and meningitis; with nosocomial infections, they possess a threat to patients with low immunity [2]. Their multidrug resistant nature poses serious healthcare issues. One of the reasons for high resistance is attributed to high efflux pump activity [3] Efflux pumps are transport proteins that extrude toxic substances including antibiotics from the bacterial cells thereby resulting in resistance [4]. Efflux pump inhibitors (EPI) have a potential use in making antibiotics more effective against MDR strains [5]. However, the well-known EPIs-carbonyl cyanide m-chlorophenylhydrazone (CCCP), $\mathrm{PA} \beta \mathrm{N}$, reserpine, berberine, and verapamil [6] are toxic in nature and cannot be used commercially. Therefore, we focused on identifying EPI from natural products.

Punica granatum (pomegranate) has historically been known to have medicinal properties and has been used in herbal medicine to treat cancer, diarrhea, diabetes, blood pressure, leprosy, dysentery, hemorrhages, bronchitis, dyspepsia, and inflammation. It is also known to have antibacterial, antifungal, and anticancer activity [7]. While the antimicrobial activity of $P$. granatum pericarp (peel and rind) has been demonstrated $[8,9]$ and its mode of action still remains unknown.
In this study, we analyzed the synergistic activity of $P$. granatum rind (PGR) extract with various classes of antibiotics against clinical MDR isolates of $K$. pneumoniae using the checkerboard assay [10]. Further, we confirmed the activity by sequential fractionation of the extract. The hexane extract which showed the best synergistic interaction was studied by gas chromatography-mass spectrometry (GC-MS) and potential compounds were identified. The ethanol extract was also shown to increase the intracellular norfloxacin accumulation, suggesting that it could be an EPI. When the sequential fraction was tested, it was found that the butanol fraction had maximum efflux inhibition activity. The identification of such EPI from natural products can lead to antibiotic combinations which are more effective against MDR isolates.

\section{METHODS}

General

The following chemicals were obtained from Sigma-Aldrich: Ciprofloxacin, norfloxacin, CCCP, magnesium sulfate, glycine hydrochloride, potassium l-lactate, and trizma hydrochloride. Resazurin, sodium hydroxide, Mueller-Hinton broth and agar, Luria Bertani broth and agar, Nutrient broth and agar and dimethyl sulfoxide were obtained from Himedia Laboratories Pvt. Ltd., Mumbai, India. Solvents-Ethanol, n-hexane, and n-butanol were obtained from Qualigens.

Bacterial isolates

About 22 MDR isolates were collected from tertiary care hospitals in Chennai. MDR nature of these isolates was clarified by 
antibiotic susceptibility testing using the antibiotics norfloxacin (NX-10 $\mu$ g), nalidixic acid (NA-30 $\mu$ g), ciprofloxacin (CF-5 $\mu$ g), amoxyclav (AMC-30 $\mu \mathrm{g}$ ), cefotaxime (CTX-30 $\mu \mathrm{g}$ ) cefepime (CPM-30 $\mu \mathrm{g}$ ), cefoxitin (Cn-30 $\mu$ g), and imipenem (IMP-10 $\mu$ g) from (Himedia Laboratories Pvt. Ltd., Mumbai, India). The results were interpreted as per the EUCAST guidelines. K. pneumoniae MTCC 432 was used as a control strain.

\section{Plant material and extraction}

The rind of $P$. granatum variety Bhagwa was obtained from M/S "Sam Agritech, Hyderabad." The product was aseptically separated and shade dried. The dried material $(1.5 \mathrm{~kg})$ was ground, sieved and macerated with ethanol in the ratio 1:3 and kept for 5 days with intermittent shaking. The extract was filtered with Whatman number 1 filter paper $(125 \mathrm{~mm})$ and collected. It was then placed in a rotary vacuum evaporator and concentrated under pressure to give $100 \mathrm{~g}$ of a viscous material. $90 \mathrm{~g}$ of this crude extract was then dissolved in water, and sequential extraction was carried out resulting in hexane, butanol and water fractions which were concentrated in rotary vacuum evaporator [11].

\section{Antibacterial activity}

The antibacterial activity was tested by determining the minimum inhibitory concentration (MIC) of the plant extract using a stock solution of the plant extract ( $50 \mathrm{mg} / \mathrm{ml}$ of $50 \%$ dimethyl sulfoxide [DMSO]) by the resazurin assay [12]. The experiment was done in triplicates and positive control (isolate $+\mathrm{LB}$ broth), negative control (LB broth), and solvent control (50\%DMSO + isolate + LB broth) were maintained. To determine the MIC of ciprofloxacin, the same experimental procedure was followed with stock solution of ciprofloxacin $(100 \mathrm{mg} / \mathrm{ml})$ added to the first well instead of the plant extract.

\section{Synergistic studies}

Synergistic interaction with ciprofloxacin was determined by checkerboard assay [13], in which 36 different combinations of plant extract and ciprofloxacin are tested to determine the best combination. The interaction between the antibiotics and plant extract was determined and quantified by calculating the fractional inhibitory concentration index (FICI) using the following formula:

\section{FIC index $=$ MIC of plant extract in combination MIC of plant extract alone \\ MIC of antibiotic in combination MIC of antibiotic alone}

Interpretation of the FICI [13]: FICI $=0.5$ synergy - joint effect is greater than sum of individual activity. FICI $>0.5$ to 4 indifference - joint effect is equal to sum of individual activity. FICI $>4$ antagonism - joint effect is less than sum of individual activity or effect of individual activity.

\section{GC-MS analysis}

GC-MS analysis of the PGR hexane extract was carried out using an AGILENT (Hewlett Packard) GC 5890, MS 5973 system. The following conditions were maintained for GC analysis. A HP- 5 ms Agilent column was used $(30 \times 0.25 \mu \mathrm{m} \mathrm{ID} \times 0.25 \mu \mathrm{mdf})$. Helium gas $99.9995 \%$ purity was used as a carrier with an injection volume of $1 \mu \mathrm{l}$ (split ratio 20) and a flow rate of $1 \mathrm{ml} /$ minute. The injector temperature was maintained at $250^{\circ} \mathrm{C}$, and the oven temperature was programed at $35^{\circ} \mathrm{C}$ (for 2 minutes), with an increase to rate $15,280^{\circ} \mathrm{C}$ for 5 minutes, lasting for a total of 35 minutes. Mass spectra were taken at $70 \mathrm{eV}$ with a scan range $40-700 \mathrm{~m} / \mathrm{z}$. the solvent cut time was 1 minute, scan speed was 2000 and the total GC/MS running time was 36 minutes. Peaks were identified according to the MS library- NIST 11

\section{Norfloxacin accumulation assay}

Efflux inhibition activity was determined by the norfloxacin accumulation assay [14]. In this assay, the norfloxacin accumulation is determined by the fluorescence of supernatants measured as EX (excitation) at $281 \mathrm{~nm}$ and EM (emission) at $440 \mathrm{~nm}$ with a Shimadzu
RF-5301pc fluorescence spectrophotometer. The experiment was carried out in triplicate.

\section{RESULTS AND DISCUSSION}

Synergistic interaction between PGR ethanol extract and ciprofloxacin

The antimicrobial activity of PGR extract is known but the mode of action is unknown. To identify the mode of action, we analyzed the synergistic activity of the ethanol extract with antibiotics. As fluoroquinolone resistance is normally associated with nosocomial $K$. pneumoniae infections [15] and efflux-mediated resistance [16], ciprofloxacin-resistant strains were chosen for our study.

Our first step was to determine the ciprofloxacin resistance of the 22 clinical K. pneumoniae strains by determining MIC. As per the EUCAST guidelines, the ciprofloxacin breakpoint MIC is $1 \mu \mathrm{g} / \mathrm{ml}$. 20 of the K. pneumoniae strains varied from high resistance $(780 \mu \mathrm{g} / \mathrm{ml})$ to less resistance $(12 \mu \mathrm{g} / \mathrm{ml})$ while two of them were found to be ciprofloxacin sensitive (Table 1). The antibacterial activity of PGR ethanol extract was determined by the MIC and was found to range from 25000 to $6250 \mu \mathrm{g} / \mathrm{ml}$. From this, we infer that on its own, a high amount of plant extract is required to inhibit bacteria. It was also noted that, even for the two strains which were ciprofloxacin sensitive, a high amount of PGR extract was required to inhibit the bacteria (Table 1). The control strain K. pneumoniae MTCC 432 was ciprofloxacin sensitive, and the MIC of PGR ethanol extract was $195 \mu \mathrm{g} / \mathrm{ml}$.

We then tested for synergy between PGR ethanol extract and ciprofloxacin by the checkerboard assay [10,13]. 36 different combinations were tested and the optimum combination of ciprofloxacin and plant extract concentration was determined. Based on an FICI, the combinations were classified as synergistic, antagonistic, and indifferent.

In 13 of the 20 strains, synergistic activity of PGR and ciprofloxacin was observed. In four of these strains, a 32-34 fold decrease in ciprofloxacin MIC was observed when in the presence of the PGR ethanol extract. Seven of the strains showed an 8-24 fold decrease and only 2 strains showed a 2-4 fold reduction of MIC. One of the strains K35 showed a 24-fold reduction of ciprofloxacin MIC in the presence of PGR making it a sensitive strain (MIC $=0.5 \mu \mathrm{g} / \mathrm{ml}$ after combining with ethanol extract). These data show that the addition of PGR ethanol extract can reduce the ciprofloxacin MIC in K. pneumoniae isolates.

Further, despite the fact that seven strains out of 20 were classified as indifferent; a fold reduction was found in the MIC of ciprofloxacin in the presence of PGR. Of these, three strains showed an 8-fold reduction, three showed a 4 -fold reduction and one showed no change.

As the ethanol extract of PGR had very good synergistic activity with ciprofloxacin (up to 34-fold reduction of MIC and re-sensitization of a resistant strain), we decided to determine whether the synergism of PGR extract was specific to ciprofloxacin or if it could interact with other antibiotics as well.

\section{Synergistic interaction with other classes of antibiotics}

Once it was determined that the PGR ethanol extract worked synergistically with ciprofloxacin and resulted in a reduction of the antibiotic MIC, we decided to study its interaction with other antibiotics.

The synergistic interaction with other classes of antibiotics such as cephalosporins, $\beta$-lactams, and aminoglycosides was determined for a representative $K$. pneumoniae strain U25 which showed a 16 -fold reduction in MIC of ciprofloxacin and is known to have high efflux activity [17].

Out of the 12 antibiotics tested, 6 of them - ciprofloxacin, levofloxacin ceftazidime, cefoxitin, meropenem and gentamicin, exhibited synergism with the ethanol extract for the strain U25 (Table 2). Ciprofloxacin showed maximum synergism with the ethanol extract showing a 
16 fold reduction, followed by cefoxitin and meropenem showing a 12 and 8 fold reduction, respectively. Levofloxacin, gentamicin, and ceftazidime were found to decrease 4 -fold in combination with ethanol extract.

In the 6 antibiotics in which indifference was noted, a 2-4 fold reduction of antibiotic was noted except for oxacillin where a 32 -fold reduction was noted. In the case of oxacillin, despite the fold reduction being so high, it is classified as indifferent as the highest concentration of plant extract is used.

This shows that while the PGR ethanol extract shows synergy over a broad spectrum of antibiotics, the highest reduction of MIC was noted in combination with ciprofloxacin.

This supports the fact that the PGR ethanol extract could have potential EPI activity.

As our objective is to identify compounds with efflux inhibition activity, we sequentially fractionated the ethanol extract with low polar hexane and highly polar butanol in a hope to extract single compounds.

\section{Synergistic extraction with the sequential plant fractions}

Once the synergistic activity of the PGR ethanol extract with ciprofloxacin was determined, sequential hexane, butanol and water fractions were obtained and were found to have antibacterial activity (data not shown). The checkerboard assay was carried out to determine if the sequential fractions as well exhibited synergism with ciprofloxacin Four representative strains were chosen for this assay, two in which, ethanol extract-antibiotic synergism activity was found (U25, K15) and two in which indifference was noted (K21, K37).

It was found that all of the sequential fractions-hexane, butanol and water, exhibited synergistic interaction with ciprofloxacin (Table 3). Even the strains K21 and K37 in which indifference was seen with the ethanol extract, synergism was seen with the sequential fractions and ciprofloxacin.

The hexane fraction showed maximum synergistic activity with ciprofloxacin. The strains U25 and K15 which showed a 16 fold MIC reduction with ethanol extract, exhibited a 32 -fold reduction with hexane fraction. An 8-32 fold reduction was seen with the water fraction while the butanol fraction showed a 2-4 fold reduction.

Table 1: Synergistic interaction between Punica granatum rind ethanol extract and ciprofloxacin

\begin{tabular}{|c|c|c|c|c|c|}
\hline \multirow[t]{2}{*}{ Strain } & \multicolumn{3}{|c|}{$\mathrm{MIC} \mu \mathrm{g} / \mathrm{ml}$} & \multirow[t]{2}{*}{ FIC } & \multirow[t]{2}{*}{ Interpretation } \\
\hline & Ciprofloxacin & PGR ethanol & Ciprofloxacin/PGR ethanol concentration & & \\
\hline K38 & 780 & 6250 & $390 / 390$ & 0.56 & Synergy \\
\hline K13 & 625 & 25000 & $78 / 6250$ & 0.37 & Synergy \\
\hline $\mathrm{U} 25$ & 390 & 12500 & $24 / 6250$ & 0.56 & Synergy \\
\hline K21 & 390 & 25000 & $97.5 / 12500$ & 0.75 & Indifference \\
\hline K3 & 390 & 25000 & $24 / 12500$ & 0.56 & Synergy \\
\hline K39 & 390 & 6250 & $12 / 3125$ & 0.53 & Synergy \\
\hline K31 & 312.5 & 12500 & $9.75 / 6250$ & 0.53 & Synergy \\
\hline K32 & 312.5 & 12500 & $9.75 / 6250$ & 0.53 & Synergy \\
\hline K15 & 312.5 & 25000 & $19.5 / 12500$ & 0.56 & Synergy \\
\hline K12 & 312 & 12500 & $78 / 3125$ & 0.50 & Synergy \\
\hline K9 & 312 & 12500 & $39 / 12500$ & 1.13 & Indifference \\
\hline K36 & 156 & 6250 & $156 / 6250$ & 2.00 & Indifference \\
\hline K29 & 156 & 12500 & $4.5 / 6250$ & 0.53 & Synergy \\
\hline K11 & 78 & 25000 & $20 / 12500$ & 0.76 & Indifference \\
\hline $\mathrm{K} 40$ & 45 & 6250 & $5.5 / 1560$ & 0.37 & Synergy \\
\hline K28 & 39 & 12500 & $4.5 / 12500$ & 1.12 & Indifference \\
\hline K37 & 12 & 6250 & $3.5 / 6250$ & 1.29 & Indifference \\
\hline K35 & 12 & 25000 & $0.5 / 12500$ & 0.54 & Synergy \\
\hline K10 & $\mathrm{S}$ & 25000 & ND & ND & ND \\
\hline K33 & $\mathrm{S}$ & 12500 & ND & ND & ND \\
\hline MTCC 432 & $\mathrm{~S}$ & 195 & ND & ND & ND \\
\hline
\end{tabular}

MIC: Minimum inhibitory concentration, FIC: Fractional inhibitory concentration where FIC of $<0.5$ is considered and $>0.5$ to 4 as indifference, PGR: Punica granatum rind, ND: Not determined, P. granatum: Punica granatum

Table 2: Synergistic interaction between Punica granatum rind ethanol extract and other antibiotics for the strain U25

\begin{tabular}{|c|c|c|c|c|c|}
\hline \multirow[t]{2}{*}{ Antibiotic } & \multicolumn{3}{|c|}{$\mathrm{MIC} \mu \mathrm{g} / \mathrm{ml}$} & \multirow[t]{2}{*}{ FIC } & \multirow[t]{2}{*}{ Interpretation } \\
\hline & AB MIC for U25 & PGR ethanol & Antibiotic/PGR ethanol concentration & & \\
\hline Ciprofloxacin & 390 & 12500 & $24 / 6250$ & 0.56 & Synergy \\
\hline Nalidixic acid & 6250 & 12500 & $3125 / 6250$ & 1.00 & Indifference \\
\hline Levofloxacin & 390 & 12500 & $97.5 / 1560$ & 0.37 & Synergy \\
\hline Norfloxacin & 6250 & 12500 & $3125 / 3125$ & 0.75 & Indifference \\
\hline Chloramphenicol & 780 & 12500 & $195 / 6250$ & 0.75 & Indifference \\
\hline Ceftazidime & 25000 & 12500 & $6250 / 3125$ & 0.50 & Synergy \\
\hline Cefotaxime & 25000 & 12500 & $12500 / 3000$ & 0.74 & Indifference \\
\hline Cefoxitin & 625 & 12500 & $50 / 6250$ & 0.58 & Synergy \\
\hline Meropenem & 62.5 & 12500 & $7.8 / 1560$ & 0.25 & Synergy \\
\hline Oxacillin & 50000 & 12500 & $1560 / 12500$ & 1.03 & Indifference \\
\hline Cloxacillin & 25000 & 12500 & $12500 / 3125$ & 0.75 & Indifference \\
\hline Gentamicin & 12500 & 12500 & $3125 / 1560$ & 0.37 & Synergy \\
\hline
\end{tabular}

to 4 as indifference, PGR: Punica granatum rind 
The strains K21 and K37 in which indifference was seen with the ethanol extract and showed synergy with all three solvent fraction resulting in a 2-4 fold reduction of ciprofloxacin MIC. The strain K37 was even made sensitive with the butanol fraction - antibiotic combination. From this, we can infer that each of the fractions has synergistic activity, but the highest fold reduction of MIC is seen in the hexane fraction. To determine the active compounds that could play a role in the synergism, GC-MS of this fraction was carried out.

\section{GC-MS analysis}

GC-MS analysis plays a major role in identifying active compounds in plant extracts [18]. The phytochemical constituents of the hexane fraction which exhibited very good synergistic activity with ciprofloxacin were analyzed by GC-MS. Eight peaks of interest indicating phytochemical constituents were observed (Fig. 1). On comparison of the spectra with the NIST database, the compounds were identified (Table 4). The results showed that the major components were linoleic acid ethyl ester and hexadecanoic acid ethyl ester with an area \% of 36.21 and 24.83 , respectively. This is followed by ethyl 9-hexadecenoate (13.45\%), n-hexadecanoic acid (7.79\%), octadecanoic acid, ethyl ester $(6.02 \%)$, eicosanoic acid, ethyl ester (1.31\%), octadecanoic acid (1.11\%), and nonadecanoic acid ethyl ester $0.94 \%$.

These are fatty acids and their esters whose antimicrobial activity has been observed [19]. These fatty acids and their esters might be interacting with the bacterial membrane to enhance the susceptibility to antibiotics. To specifically find out if the PGR extracts had an effect of the efflux pumps, we tested the fractions by the norfloxacin accumulation assay [14].

\section{EPI activity}

The efflux inhibition activity of PGR ethanol extract and the hexane, butanol and water fraction was analyzed by the norfloxacin accumulation assay [14] using a representative $K$. pneumoniae isolate U25 which is known to have high efflux activity [17]. A well-known EPI CCCP that dissipates the proton gradient was used as a control [20]. In this assay, the amount of norfloxacin accumulated within the cell is estimated after the addition of an EPI. It was found that on addition of PGR ethanol extract a high intracellular accumulation of norfloxacin was seen. When compared to the standard CCCP, the PGR ethanol extract was found to lead to higher intracellular norfloxacin accumulation (Fig. 2)

When the sequential fractions were tested, it was found that addition of the butanol fraction resulted in higher norfloxacin accumulation. Despite the fact that the hexane fraction showed maximum synergistic activity, it was found that the butanol fraction had a much higher EPI activity. These data suggest that the components of PGR butanol fraction can inhibit the efflux pumps, and therefore, one or more components could be a potential EPI.

Studies have been carried out with the PGR extract by disc diffusion method and MIC [921] against MTCC strains (using $1 \mathrm{~K}$. pneumoniae isolate) and methicillin resistant Staphylococcus aureus, respectively. Our report analyses the synergistic activity of PGR extract with antibiotics using the checkerboard method against 22 clinical isolates of MDR K. pneumoniae.

Studies have also found that $P$. granatum has the ability to inhibit NorA efflux pumps in Staphylococcus aureus [22]. Taken together with our data, it is likely that PGR butanol fraction inhibits the efflux pumps in $K$. pneumoniae. Further analysis of the compounds in the butanol fraction is in progress.

P. granatum tannins punicalagin, punicalin, and ellagic acid have been found to specifically block the HCV NS3/4A protease activity in vitro and have been found to be bioavailable [23]

Table 3: Synergistic interaction between ethanol extract, sequential fractions in hexane, butanol and water with ciprofloxacin

\begin{tabular}{|c|c|c|c|c|c|c|c|c|c|c|}
\hline Culture & CIP & $\begin{array}{l}\text { PGR } \\
\text { extract }\end{array}$ & $\begin{array}{l}\text { CIP+PGR } \\
\text { butanol } \\
\text { fraction }\end{array}$ & Activity & $\begin{array}{l}\text { CIP+PGR } \\
\text { hexane fractionMIC }\end{array}$ & $\begin{array}{l}\text { Activity } \\
\mathrm{C} \text { in } \mu \mathrm{g} / \mathrm{ml}\end{array}$ & $\begin{array}{l}\text { CIP+PGR } \\
\text { water fraction }\end{array}$ & Activity & $\begin{array}{l}\text { CIP+PGR } \\
\text { ethanol extract }\end{array}$ & Activity \\
\hline U25 & 390 & 25000 & $97.5 / 1560$ & $\mathrm{~S}$ & $12 / 12500$ & S & $48.5 / 780$ & $\mathrm{~S}$ & $24 / 6250$ & S \\
\hline K21 & 390 & 25000 & $97.5 / 6250$ & S & $120 / 6250$ & S & $156 / 3120$ & $\mathrm{~S}$ & $97.5 / 12500$ & I \\
\hline K37 & 12 & 25000 & $\mathrm{~S}$ & S & $6 / 1560$ & S & $6 / 1560$ & S & $3.5 / 6250$ & I \\
\hline
\end{tabular}

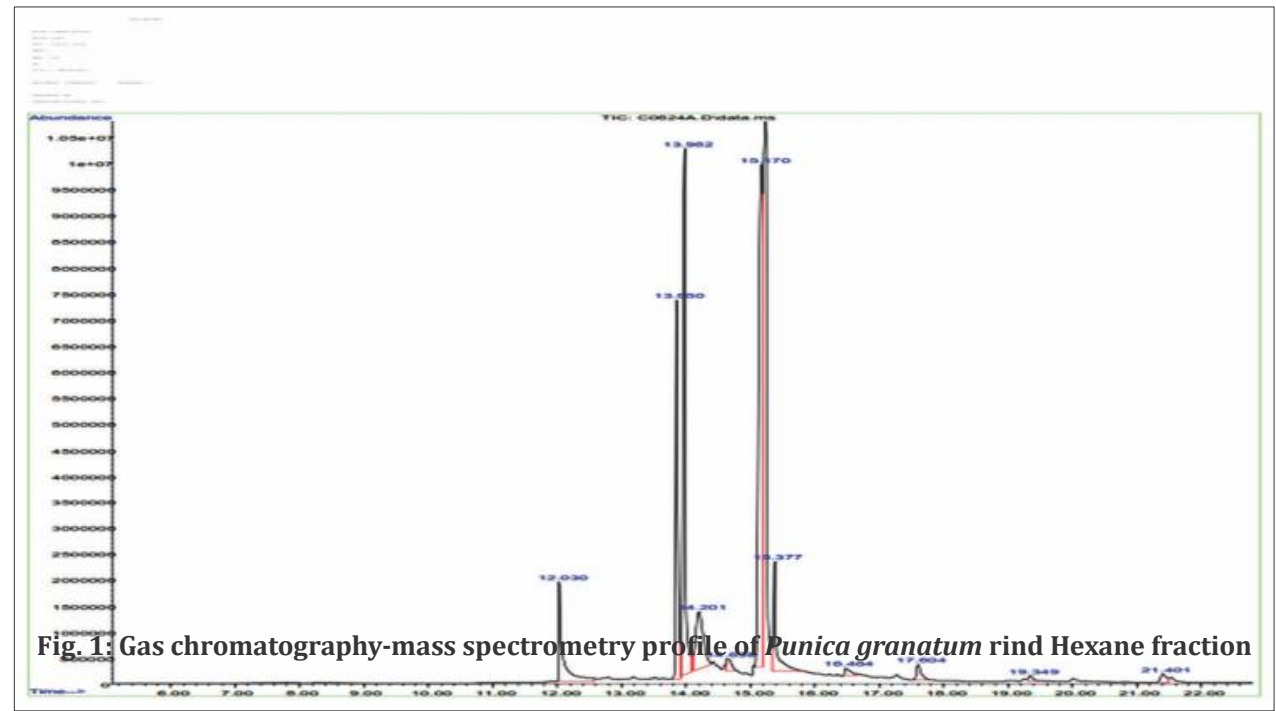


Table 4: Compounds from PGR hexane fraction identified by GC-MS analysis

\begin{tabular}{lll}
\hline Retention time & Name of compound & Area \% \\
\hline 13.85 & Ethyl 9-hexadecenoate & 13.45 \\
13.982 & Hexadecanoic acid, ethyl ester & 24.83 \\
14.201 & n-Hexadecanoic acid & 7.79 \\
14.639 & Octadecanoic acid & 1.11 \\
15.17 & Linoleic acid ethyl ester & 36.21 \\
15.377 & Octadecanoic acid, ethyl ester & 6.02 \\
17.604 & Eicosanoic acid, ethyl ester & 1.31 \\
21.401 & Nonadecanoic acid, ethyl ester & 0.94 \\
\hline
\end{tabular}

PGR: Punica granatum rind, GC-MS: Gas chromatography-mass spectrometry

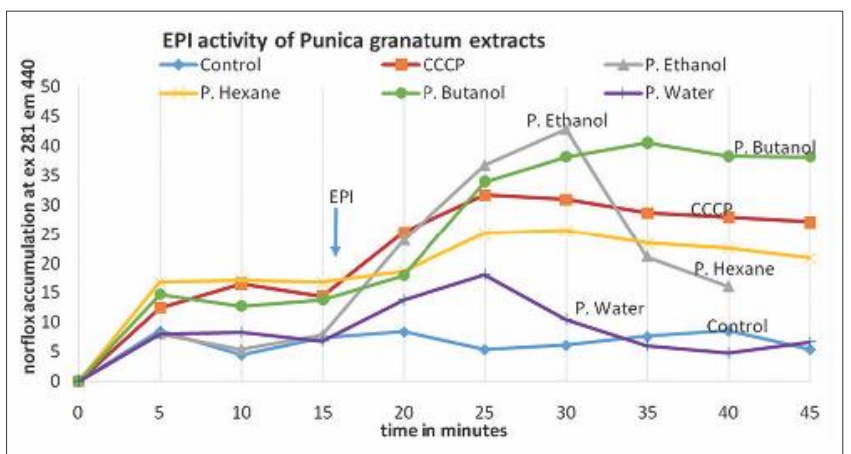

Fig. 2: Efflux inhibition activity of Punica granatum rind ethanol extract and sequential fractions

Our study shows that PGR extracts have synergistic activity with ciprofloxacin and the butanol soluble fraction from PGR specifically inhibits efflux pump activity. Analyzing leads from traditional knowledge to identify compounds that can be used as adjuvant for antimicrobial therapy is an important field of research. The use of such synergistic compounds can re-sensitize resistant isolates and make antimicrobial therapy more efficient.

\section{CONCLUSION}

In this report, we show that PGR ethanol extract exhibits a consistent reduction of antibiotic MIC. Even in the strains that the plant-antibiotic interaction was classified as indifferent, a fold reduction of the antibiotic is still seen. This synergistic interaction was seen with a broad spectrum of antibiotics such as ciprofloxacin, levofloxacin, ceftazidime, cefoxitin, meropenem, and gentamicin. To identify the individual compounds with EPI activity, sequential extraction of the PGR ethanol extract was done. Of the sequential fractions, the hexane fraction was found to have the most synergistic activity with ciprofloxacin, resulting in a 32 -fold reduction of MIC. The mode of action of PGR ethanol and sequential fractions were also studied and it was found that addition of PGR extract results in increased intracellular norfloxacin accumulation. In this case, it was found that the PGR butanol fraction had the best EPI activity. This indicates one or more components of the PGR butanol fraction could be a potential EPI.

\section{ACKNOWLEDGMENT}

We would like to thank Dr. K Balakrishna (Emeritus Scientist, Entomology Research Institute, Loyola College, Chennai - 34) for helpful discussions and CVR LABS Pvt Ltd, Chennai - 15, for the GCMS analysis.

\section{REFERENCES}

1. Nikaido H. Multidrug resistance in bacteria. Annu Rev Biochem 2009;78(3):119-46.
2. Podschun R, Ullmann U. Klebsiella spp. As nosocomial pathogens: Epidemiology, taxonomy, typing methods, and pathogenicity factors. Clin Microbiol Rev 1998;11(4):589-603.

3. Padilla E, Llobet E, Doménech-Sánchez A, Martínez-Martínez L, Bengoechea JA, Albertí S. Klebsiella pneumoniae AcrAB efflux pump contributes to antimicrobial resistance and virulence. Antimicrob Agents Chemother 2010;54(1):177-83.

4. Webber MA, Piddock LJ. The importance of efflux pumps in bacterial antibiotic resistance. J Antimicrob Chemother 2003;51(1):9-11.

5. Mehta J, Jandaik S. Evaluation of phytochemicals and synergistic interaction between plant extracts and antibiotics for efflux pump inhibitory activity against Salmonella enterica serovar Typhimurium strains. Int J Pharm Pharm Sci 2016;8(10):217-23.

6. Stavri M, Piddock LJ, Gibbons S. Bacterial efflux pump inhibitors from natural sources. J Antimicrob Chemother 2007;59(6):1247-60.

7. da Silva JA, Rana TS, Narzary D, Verma N, Meshram DT, Ranade S. Pomegranate biology and biotechnology: A review. Sci Hortic Amsterdam 2013;160(8):85-107.

8. Dahham SS, Ali MN, Tabassum H, Khan M. Studies on antibacterial and antifungal activity of pomegranate (Punica granatum L.). Am J Agric Environ Sci 2010;9(3):273-81.

9. Rathinamoorthy R, Udayakumar S, Thilagavathi G. Antibacterial efficacy analysis of Punica granatum L. Leaf, rind and Terminalia chebula fruit extract treated cotton fabric against five most common human pathogenic bacteria. Int J Pharm Life Sci 2011;2(10):1147-53.

10. Chanda S, Rakholiya K. Combination therapy: Synergism between natural plant extracts and antibiotics against infectious diseases. In: Méndez-Vilas A, editor. Science against Microbial Pathogens: Communicating Current Research and Technological Advances. Spain: Formatex Research Center; 2011. p. 520-9.

11. Shukla N, Kumar M, Akanksha, Ahmad G, Rahuja N, Singh AB, et al. Tectone, a new antihyperglycemic anthraquinone from Tectona grandis leaves. Nat Prod Commun 2010;5(3):427-30.

12. Sarker SD, Nahar L, Kumarasamy Y. Microtitre plate-based antibacterial assay incorporating resazurin as an indicator of cell growth, and its application in the in vitro antibacterial screening of phytochemicals. Methods 2007;42(4):321-4.

13. Stefanovic O, Stankovic MS, Comic L. In vitro antibacterial efficacy of Clinopodium vulgare L. Extracts and their synergistic interaction with antibiotics. J Med Plants Res 2011;5(17):4074-9.

14. Li L, He Z, Pandey GK, Tsuchiya T, Luan S. Functional cloning and characterization of a plant efflux carrier for multidrug and heavy metal detoxification. J Biol Chem 2002;277(7):5360-8

15. Lautenbach E, Fishman NO, Bilker WB, Castiglioni A, Metlay JP, Edelstein $\mathrm{PH}$, et al. Risk factors for fluoroquinolone resistance in nosocomial Escherichia coli and Klebsiella pneumoniae infections. Arch Intern Med 2002;162(21):2469-77.

16. Poole K. Efflux-mediated resistance to fluoroquinolones in gramnegative bacteria. Antimicrob Agents Chemother 2000;44(9):2233-41.

17. Rafiq Z, Sam N, Vaidyanathan R. Whole genome sequence of Klebsiella pneumoniae U25, a hypermucoviscous, multidrug resistant, biofilm producing isolate from India. Mem Inst Oswaldo Cruz 2016;111:144-6.

18. Thangavel A, Senthilkumar B, Vijayalakshmi, Duraisamy S. Phytochemical screening, gas chromatography-mass spectrometry (Gc-Ms) analysis of phytochemical constituents and antibacterial activity of coriandrum sativum (1) seeds. Int. J. Pharm. Pharm. Sci. 2015;7(9):153-9

19. Kabara JJ, Swieczkowski DM, Conley AJ, Truant JP. Fatty acids and derivatives as antimicrobial agents. Antimicrob Agents Chemother 1972;2(1):23-8.

20. Cho H, Oh Y, Park S, Lee Y. Concentration of CCCP Should be optimized to detect the efflux system in quinolone-susceptible Escherichia coli. J Microbiol 2001;39(1):62-6.

21. Aqil F, Khan MS, Owais M, Ahmad I. Effect of certain bioactive plant extracts on clinical isolates of $\beta$-lactamase producing methicillin resistant Staphylococcus aureus. J Basic Microbiol 2005;45(2):106-14.

22. Braga LC, Leite AA, Xavier KG, Takahashi JA, Bemquerer MP, Chartone-Souza E, et al. Synergic interaction between pomegranate extract and antibiotics against Staphylococcus aureus. Can J Microbiol 2005;51(7):541-7.

23. Reddy BU, Mullick R, Kumar A, Sudha G, Srinivasan N, Das S. Small molecule inhibitors of HCV replication from pomegranate. Sci Rep 2014;4:5411. 